\title{
Influência da dificuldade de inseminação, temperamento e cortisol plasmático sobre a taxa de concepção de vacas e novilhas da raça Nelore inseminadas em tempo fixo
}

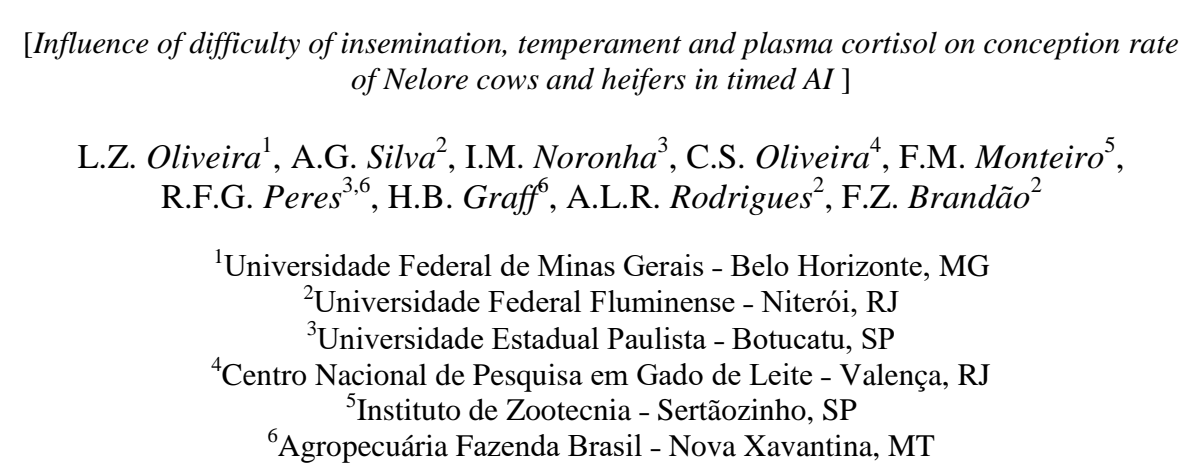




\section{INTRODUÇÃO}

Embora sejam notórios os avanços a partir do desenvolvimento comercial da inseminação artificial em tempo fixo (IATF), os diversos estudos envolvendo a utilização de diferentes protocolos apontam índices médios de concepção ao redor de 40 a $65 \%$ para gado de corte (Baruselli et al., 2004; Meneghetti et al., 2009; Peres et al., 2009; Bó e Baruselli, 2014; Ferraz Júnior et al., 2016).

Inúmeras causas podem ser apontadas para justificar a amplitude de resultados dos programas reprodutivos. Dentre elas, destacamse o escore de condição corporal (ECC) da fêmea (Meneghetti et al., 2009), o padrão racial e/ou a categoria animal (Peres et al., 2009; Sartori et al., 2016), o tamanho do folículo dominante, o grau de expressão de estro e/ou o tamanho do corpo lúteo (CL) formado (Perry et al., 2007; Mesquita et al., 2014). Adicionalmente, o touro, a partida e/ou o manejo de palhetas também podem influenciar a taxa de concepção (TC) da IATF (Oliveira et al., 2012). Outro fator que pode interferir nos índices reprodutivos é o nível de estresse do animal (Cooke et al., 2011; Maziero et al., 2012; Cooke et al., 2017; Narayan e Parisella, 2017).

Diferentes parâmetros têm sido utilizados para avaliar subjetivamente o temperamento, os quais se baseiam no comportamento e/ou na demonstração de agressividade do animal (Cooke et al., 2011; Sant'Anna et al., 2015; Valente et al., 2015). Maior reatividade do animal determina maior custo de manejo por aumentar a demanda de funcionários, acrescentar tempo necessário para a execução do serviço, diminuir a segurança de trabalho, reduzir a vida útil do curral e, ainda, aumentar o nível de estresse dos animais em geral (Paranhos da Costa et al., 2015). Atividades que consistem em manejos consecutivos no curral podem acarretar respostas fisiológicas e comportamentais típicas de comprometimento do bem-estar, aumentando a reatividade do gado (Rueda et al., 2015), principalmente quando realizadas de forma equivocada (Grandin, 1993; Paranhos da Costa et al., 2015).

A resposta gerada pelo organismo sob situação de estresse envolve a secreção de glicocorticoides e progesterona pelas glândulas adrenais (desencadeada pelo eixo hipotalâmicohipofisário-adrenal), afetando diretamente a fisiologia reprodutiva do animal (Bage et al., 2000; Dobson e Smith, 2000). O aumento da secreção de progesterona pela adrenal (associado ao aumento da secreção de cortisol) pode interferir negativamente na fertilidade, e a importância dessa influência pode variar de acordo com o dia do protocolo de sincronização da ovulação em que ocorrer essa variação hormonal (Cooke et al., 2011). Estudos em bovinos mostraram que os efeitos negativos provocados pelo estresse inibiram o pico préovulatório de LH e reduziram a qualidade dos embriões produzidos (Macedo et al., 2011; Maziero et al., 2012). Adicionalmente, alterações em parâmetros fisiológicos de vacas Nelore causadas por situações de estresse diminuíram a probabilidade de gestação após IATF (Rueda et al., 2015; Cooke et al., 2017).

Além do temperamento do animal, outro fator que poderia afetar a fertilidade durante a realização da IATF é a dificuldade de se completar o procedimento de inseminação, visto que algumas fêmeas bovinas podem apresentar cérvix mais tortuosa e/ou estreita que outras. Porém, esse aspecto não tem sido levado em consideração nas avaliações de resultados de programas de IA. Assim, o presente estudo teve por objetivo avaliar a influência do nível de estresse e do grau de dificuldade de inseminação de vacas e novilhas da raça Nelore submetidas à IATF, de modo a verificar se esses fatores interferem nas taxas de concepção a campo desses programas reprodutivos.

\section{MATERIAL E MÉTODOS}

O presente projeto foi aprovado pelo Comitê de Ética no Uso de Animais (Ceua) da Universidade Federal Fluminense (Protocolo Ceua UFF 884/2016). Este estudo foi conduzido durante estação de monta 2016/2017, em uma fazenda comercial de gado de corte, localizada no município de Nova Xavantina, MT. Foram coletados os dados de inseminações para primeiro serviço de 165 fêmeas bovinas da raça Nelore (Bos indicus), sendo 72 novilhas (24 a 26 meses, peso entre 300 e $330 \mathrm{~kg}$ ) e 93 vacas pluríparas (50 a 70 meses, peso entre 360 e $420 \mathrm{~kg}$ ), com 50 dias pós-parto. Os animais foram mantidos em pasto composto por Brachiaria 
brizantha, suplementados com sal mineral e livre acesso à água.

As fêmeas receberam o protocolo de IATF descrito por Peres et al. (2009). Esse iniciou-se (D0) com as vacas recebendo um dispositivo intravaginal (de segundo uso) contendo progesterona (P4; CIDR, Zoetis Saúde Animal, São Paulo, Brasil) e uma injeção intramuscular (IM) de 2,0mg de benzoato de estradiol (BE; Gonadiol, Zoetis Saúde Animal, São Paulo, Brasil). Sete dias após (D7), os animais receberam $25 \mathrm{mg}$ (IM) de prostaglandina F2 $\alpha$ (Dinoprost trometamina; Lutalyse Zoetis Saúde Animal, São Paulo, Brasil). O dispositivo de P4 foi removido nove dias após (D9), quando os animais receberam injeções de 300UI de gonadotrofina coriônica equina (eCG; Novormon 5000 UI, Zoetis Saúde Animal, São Paulo, Brasil) e 0,5mg de cipionato de estradiol (ECP, Zoetis Saúde Animal, São Paulo, Brasil), ambos pela via IM. Dois dias após a remoção do dispositivo (ou seja, no D11), os animais foram inseminados (IATF). Esse mesmo protocolo foi utilizado nas novilhas, sendo os implantes de P4 (CIDR, Zoetis Saúde Animal, São Paulo, Brasil) de terceiro uso.

Em todas essas fêmeas, foram utilizadas doses de sêmen de quatro touros da raça Angus, aleatoriamente distribuídos nos dias de IA, de forma equiparada. Para a realização das inseminações, dois experientes inseminadores intercalaram-se entre si a cada animal que passava pelo tronco de contenção (brete). Todos os procedimentos de campo envolvendo este estudo foram realizados em um curral bem estruturado e coberto. O escore de condição corporal (ECC) foi aferido visualmente, variando de 1 a 5 , sendo 1 a nota aferida a animais muito magros e 5 a nota aferida a animais obesos. No presente experimento, foram utilizados apenas os dados das vacas e novilhas que apresentaram ECC acima de 2,75 no D9.

No dia da retirada do implante de P4 (D9), foram cautelosamente anotados diversos dados relativos a cada vaca ou novilha durante sua passagem pelo brete. Esses dados incluíram o número do animal, o ECC, a nota de reatividade/ temperamento (NTe) e o tempo da saída do brete (TSB). Além disso, foi coletado sangue das novilhas para posterior dosagem de cortisol. No dia da IATF (D11), foram anotados número do animal, ECC, inseminador, touro, partida de sêmen, NTe e TSB. Ademais, cada animal recebeu uma nota pela DifIA (aferida pelo inseminador), e o tempo para se completar cada inseminação (tempo de IA) foi cronometrado.

A NTe consistiu na classificação visual por observação do comportamento/reatividade do animal, de acordo com as características de perturbação dele, durante seu período de permanência no tronco de contenção. Essa avaliação foi aferida para todos os animais pelo mesmo avaliador, classificada de 1 a 5 , com base nos trabalhos de Paranhos da Costa et al. (2015) e Valente et al. (2015), modificada conforme descrito a seguir:

NTe1 = sem comportamento de estresse = animal parado sem expressar incômodo durante atividade no brete (extremamente tranquilo, sem movimentos bruscos de cauda, orelhas e cabeça; permite total proximidade do observador sem alterar comportamento);

NTe2 = comportamento de estresse baixo = animal calmo ou pouco ativo durante atividade no brete (pouca movimentação; animal tranquilo, mas alerta; parado na maior parte do tempo de observação; permite certa proximidade do observador);

NTe3 = comportamento de estresse moderado $=$ animal inquieto durante atividade no brete (movimentação frequente, mas pouco vigorosa; animal agitado com movimento corporal indicativo de incômodo durante maior parte do tempo de observação);

NTe4 = comportamento de estresse considerável $=$ movimentação intensa durante atividade no brete (movimento constante da cauda, constante tentativa de fuga; animal tenso, debate-se vigorosamente; não permite grande proximidade do observador);

NTe $5=$ comportamento de estresse alto $=$ animal muito estressado (perturbado), dificultando o serviço no brete (movimentação constante e muito vigorosa; animal pula, levantando os membros do piso, o que causa grande ruído. Tenta atacar o observador).

O TSB foi obtido por meio de um cronômetro digital. A contagem do cronômetro foi iniciada a partir da abertura do brete (que permitiu a saída do animal da estrutura de contenção) e encerrada assim que a fêmea bovina atravessou um ponto fixo previamente definido, posicionado a uma 
distância de dois metros após a porta de saída do brete. A passagem da cabeça do animal foi utilizada como referência para finalizar a cronometragem (Cooke et al., 2011). O TSB foi aferido sempre pelo mesmo técnico.

Adicionalmente, a nota de dificuldade de inseminação (DifIA) foi definida pelo próprio inseminador no dia da IATF (D11), sendo avaliada de 1 a 3 . A nota 1 para DifIA (DifIA1) correspondeu ao procedimento de transposição da cérvix para a realização da IA com extrema facilidade (ou seja, ausência de dificuldade para se completar a inseminação). A nota 2 para DifIA (DifIA2) correspondeu ao procedimento de transposição da cérvix com dificuldade moderada para se completar a inseminação, e a nota DifIA3 correspondeu a um procedimento de transposição da cérvix com alto grau de dificuldade para se completar a inseminação. Durante esse momento de realização da IA propriamente dita, o tempo necessário para se completar a inseminação (tempo de IA) também foi aferido com cronômetro zerado. A contagem do cronômetro foi iniciada no momento da inserção do aplicador de sêmen no vestíbulo vaginal do animal. Tal cronometragem foi finalizada no momento em que o aplicador foi retirado do trato reprodutivo da fêmea (já sem a presença de sêmen em seu interior), sendo completamente removido do limite definido pela vulva do animal.

Aos 40 dias após as inseminações, a gestação foi diagnosticada com ultrassonografia transretal, por meio de transdutor linear $(7,5 \mathrm{MHz})$. A observação de uma vesícula embrionária com embrião viável (presença de batimento cardíaco) foi o critério utilizado para confirmação da prenhez. Dessa forma, avaliou-se a taxa de concepção (TC) do presente estudo, em que se calculou a proporção de animais gestantes sobre o total das fêmeas inseminadas.

As coletas de sangue para dosagem de cortisol foram realizadas por punção da veia coccígea, utilizando-se tubo contendo EDTA $10 \%(5 \mathrm{~mL})$ do sistema Vacutainer (BD Diagnostics, São Paulo, Brasil). Para dosagem das concentrações circulantes de cortisol, o plasma foi obtido por meio da centrifugação do sangue (900 $x \mathrm{~g}$ por $15 \mathrm{~min})$ e submetido ao rápido congelamento ($20^{\circ} \mathrm{C}$ ) em tubos plásticos, que foram mantidos a essa temperatura até o momento da realização das dosagens.

A coleta de sangue foi realizada no dia da remoção do implante de P4 (D9; antes da retirada do dispositivo), em todas as novilhas do experimento. Das amostras de plasma sanguíneo das 72 novilhas disponíveis para estudo, amostras de 44 animais foram utilizadas, de modo a excluir amostras de animais em que foi observado algum grau de dificuldade de inseminação (DifIA2 ou DifIA 3). Assim, todas as fêmeas em que se realizou a dosagem de cortisol $(n=44)$ encontravam-se na categoria DifIA 1. As concentrações plasmáticas de cortisol foram determinadas pela técnica de radioimunoensaio de fase sólida, utilizando-se kit comercial (ImmuChemTM Coated Tube, MP Biomedicals, LLC Diagnostic Division, Solon, $\mathrm{OH}$, EUA). Todos os valores determinados encontravam-se dentro dos limites da curva. O coeficiente de variação intraensaio foi de $8 \%$.

A TC foi analisada usando-se regressão logística pelo SAS (SAS Inst. Inc., Cary, NC, EUA). As variáveis consideradas foram ECC, inseminador, touro, partida, Nte no D9 e NTe no D11, TSB D9 e TSB D11, DifIA e tempo de IA. Diferenças estatísticas para cada variável foram analisadas por regressão logística, utilizando-se os métodos dos quadrados mínimos (Least squares means; LS Means) do procedimento GLIMMIX do SAS (Statistical Analysis System, versão 9.2 - 2001). Ainda, as variáveis (médias \pm desvio-padrão) foram comparadas entre si por análise de variância (teste de Tukey). Os resultados também foram submetidos à correlação de Pearson. Em todas as análises, estabeleceu-se grau de significância $5 \%$.

\section{RESULTADOS E DISCUSSÃO}

A TC total foi $40 \%$ (66/165), sendo $36 \%$ (33/93) para as vacas pluríparas e $46 \%$ (33/72) para as novilhas $(\mathrm{P}=0,1242)$. A dificuldade de inseminação foi um fator que pareceu influenciar a TC quando os dados de vacas e novilhas foram analisados em conjunto, visto que uma tendência para maior $(\mathrm{P}=0,082) \mathrm{TC}$ foi observada nos animais em que não existiu dificuldade de inseminação (DifIA 1; TP=42\%, n=143) em comparação aos animais que apresentaram moderada ou alta dificuldade de IA (DifIA $2+$ DifIA3; $\mathrm{TP}=27 \%, \quad \mathrm{n}=22)$. A TC $(59 \%)$ 
das novilhas menos reativas (cortisol $4,12 \pm 1,12 \mathrm{ng} / \mathrm{mL} ; \quad \mathrm{NTe}=3,2 \pm 0,6 ; \quad \mathrm{n}=22)$ não diferiu estatisticamente $(\mathrm{P}>0,05)$ da TC $(41 \%)$ das mais agitadas (cortisol $=7,76 \pm 1,33 \mathrm{ng} / \mathrm{mL}$; $\mathrm{NTe}=3,82 \pm 0,79 ; \mathrm{n}=22$ ).

Na América do Sul, o uso de protocolos à base de estrógenos e $\mathrm{P} 4$ corresponde ao tratamento mais utilizado para a IATF de gado Bos indicus (Baruselli et al., 2004; Meneghetti et al., 2009; Bó e Baruselli, 2014; Ferraz Júnior et al., 2016).
Ao revisarem resultados de protocolos de IATF semelhantes ao do presente estudo, Sá Filho e Vasconcelos (2011) relataram que categoria animal, ECC, touro e inseminador são alguns dos diversos fatores que podem afetar a taxa de prenhez em novilhas e vacas de corte. No presente estudo, porém, não se observou efeito na TC desses fatores $(\mathrm{P}>0,05)$ para nenhuma categoria animal, conforme demonstrado na Tab. 1 .

Tabela 1. Taxa de concepção (TC) de fêmeas da raça Nelore de acordo com ECC, inseminador e touro utilizado

\begin{tabular}{ccccccccc}
\hline & \multicolumn{2}{c}{ ECC $(1$ a 5) } & \multicolumn{2}{c}{ Inseminador } & \multicolumn{3}{c}{ Touro } \\
\hline Categoria & 2,75 a 3,25 & 3,50 a 4,00 & 1 & 2 & 1 & 2 & 3 & 4 \\
Vaca & $37 \%$ & $32 \%$ & $36 \%$ & $35 \%$ & $26 \%$ & $35 \%$ & $57 \%$ & $26 \%$ \\
& $(26 / 71)$ & $(7 / 32)$ & $(16 / 44)$ & $(17 / 49)$ & $(6 / 23)$ & $(9 / 26)$ & $(12 / 21)$ & $(6 / 23)$ \\
Novilha & $50 \%$ & $42 \%$ & $47 \%$ & $44 \%$ & $53 \%$ & $36 \%$ & $48 \%$ & $44 \%$ \\
& $(16 / 32)$ & $(17 / 40)$ & $(18 / 38)$ & $(15 / 34)$ & $(8 / 15)$ & $(4 / 11)$ & $(10 / 21)$ & $(11 / 25)$ \\
Total & $41 \%$ & $39 \%$ & $41 \%$ & $38 \%$ & $37 \%$ & $35 \%$ & $52 \%$ & $35 \%$ \\
& $(42 / 103)$ & $(24 / 62)$ & $(34 / 82)$ & $(32 / 83)$ & $(14 / 38)$ & $(13 / 37)$ & $(22 / 42)$ & $(17 / 48)$ \\
\hline
\end{tabular}

ECC: escore de condição corporal avaliado no dia da remoção do implante de P4.

Não foi observada significância $(\mathrm{P}<0,05)$ para nenhuma das variáveis sobre a TC.

A ausência de efeito da condição corporal sobre a TC do presente estudo se deve ao fato de que foram utilizadas apenas vacas e novilhas com ECC acima de 2,75 (e $\leq 4,00)$, fator ainda favorecido pelo emprego do eCG nos protocolos, o qual beneficia principalmente a fertilidade de animais mais magros (Dias et al., 2013; Bó e Baruselli, 2014). Adicionalmente, as inseminações deste trabalho foram todas realizadas sempre pelos mesmos dois experientes inseminadores. Com relação à ausência de diferença estatística para TC entre touros, não se pode excluir a possibilidade de que ela se deva ao reduzido número de animais do presente experimento.

Menchaca et al. (2013) também não observaram diferença na TC para vacas $(65,4 \% ; n=228)$ em comparação a novilhas $(45,9 \% ; \mathrm{n}=381)$, em protocolos à base de $\mathrm{P} 4$ com o uso de eCG, tanto para animais em anestro quanto para animais com CL no início do protocolo. A utilização do eCG, incorporado ao final dos protocolos de IATF, aumenta a taxa de ovulação e o diâmetro do folículo pré-ovulatório em animais Bos indicus em anestro e/ou com baixo ECC, melhorando a taxa de concepção deles (Baruselli et al., 2004; Bó e Baruselli, 2014). Além disso, o protocolo utilizado no presente trabalho consiste na administração da $\mathrm{PGF}_{2 \alpha}$ dois dias antes da retirada do implante de $\mathrm{P} 4$. Estudos anteriores (Peres et al., 2009; Mesquita et al., 2014) demonstraram que essa estratégia aumenta o tamanho do folículo ovulatório e a taxa de prenhez, ainda que acarrete um manejo a mais do gado no curral.

Embora a utilização da IATF seja uma ferramenta de grande auxílio na praticidade dos programas reprodutivos, há de se ponderar que a necessidade de manejos consecutivos de um mesmo lote de bovinos no curral (para aplicação desses protocolos hormonais) pode acarretar respostas fisiológicas e comportamentais (Cooke et al., 2011; Maziero et al., 2012; Paranhos da Costa et al., 2015). O aumento da reatividade e o comprometimento do bem-estar são mais evidentes em animais com temperamento mais sanguíneo (Silveira et al., 2006). Essas reações podem ser ainda agravadas quando o manejo é realizado de forma equivocada, por pessoas maltreinadas e/ou em currais com infraestrutura inadequada (Grandin, 1993; Hemsworth et al., 2000). A adequação de práticas de manejo é uma das principais formas de se contornar o problema da alta reatividade do gado de corte (Silveira et al., 2006). 
A propriedade em que o presente estudo foi realizado tem histórico de investimentos em treinamentos (e cursos de atualizações) dos funcionários sobre boas práticas de manejo que visam ao bem-estar dos animais (manejo racional). Adicionalmente, o temperamento é um critério utilizado como fator de descarte para os animais da propriedade em que o estudo foi conduzido, de modo que vacas muito agressivas não são mantidas para a estação de monta seguinte. Confirmando essa informação, observou-se no D9 um percentual de apenas $5,5 \%$ (4/72) de novilhas NTe5 e 2,0\% (2/93) de vacas NTe5. No D11, foi observado um percentual de apenas $11 \%(8 / 72)$ de novilhas NTe5 e 2\% (2/93) de vacas NTe5. Nesse sentido, diversos autores relatam que a seleção para animais mais calmos garante facilidade no manejo da propriedade e melhora nos índices produtivos e reprodutivos nos anos sucessivos (Hemsworth et al., 2000; Burrow e Prayaga, 2004; Maziero et al., 2012; Cooke et al., 2017).

Um dos métodos menos subjetivos da nota de temperamento é o teste de velocidade da saída do brete (ou "flight speed"), realizado logo após a soltura dos animais de uma situação em que estavam contidos isoladamente (Burrow e Prayaga, 2004). Semelhantemente, no presente estudo, avaliou-se o tempo de saída do brete (TSB) como a aferição numérica (segundos) para o animal cruzar um ponto fixo. Em ambas as passagens (D9 e D11), animais NTe2 saíram mais lentamente $(\mathrm{P}<0,001)$ do brete do que animais NTe3, e estes mais lentamente $(\mathrm{P}<0,001)$ que animais NTe4-5. A média de TSB (vacas e novilhas) no D9 foi de 03:58 $\pm 01: 38 \mathrm{~s}$ para animais $\mathrm{NTe} 2$ ultrapassarem o marco de $2 \mathrm{~m}$ de distância $(\mathrm{n}=33), \quad 03: 00 \pm 1: 11 \mathrm{~s}$ para animais NTe3 $(\mathrm{n}=82)$ e 02:34 $\pm 01: 05 \mathrm{~s}$ para animais NTe4-5 ( $\mathrm{n}=50)$. No D11, a média geral (vacas e novilhas) de TSB foi de 04:20 $\pm 01: 08$ s para NTe2 $(\mathrm{n}=27), \quad 03: 30 \pm 1: 16 \mathrm{~s}$ para NTe3 $(\mathrm{n}=63)$ e 01:59 $\pm 01: 25$ s para NTe4-5 $(n=75)$.

Pesquisas anteriores demonstraram que as respostas comportamentais e fisiológicas associadas ao temperamento de excitação podem prejudicar a eficiência reprodutiva das vacas $B$. indicus (Cooke et al., 2011). Porém, no presente trabalho, não foi observado efeito $(\mathrm{P}>0,05)$ de temperamento sobre a TC no D9, seja para pluríparas $(\mathrm{NTe} 2=36 \%, \mathrm{n}=33 ; \mathrm{NTe} 3=38 \%$, $\mathrm{n}=44 ; \quad \mathrm{NTe} 4+\mathrm{NTe} 5=25 \%, \mathrm{n}=16 ;$ Fig. 1), novilhas $(\mathrm{NTe} 3=50 \%, \mathrm{n}=38$; NTe4+NTe $5=41 \%$, $\mathrm{n}=34$; Fig. 1) ou ambas as categorias avaliadas em conjunto (NTe2=36\%, n=33; NTe3 $=44 \%$, $\mathrm{n}=82$; NTe4-5=36\%, $\mathrm{n}=50$ ), nem no D11, tanto para pluríparas $(\mathrm{NTe} 2=30 \%, \mathrm{n}=27$; $\mathrm{NTe} 3=43 \%$, $\mathrm{n}=46 ; \quad \mathrm{NTe} 4-5=25 \%, \quad \mathrm{n}=20), \quad$ novilhas ( $\mathrm{NTe} 3=47 \%, \mathrm{n}=17 ; \mathrm{NTe} 4-5=45 \%, \mathrm{n}=55)$ como para ambas as categorias $(\mathrm{NTe} 2=30 \%, \mathrm{n}=27$; $\mathrm{NTe} 3=44 \%, \mathrm{n}=63$; NTe4-5=40\%, $\mathrm{n}=75$ ).

Quando os dados de cortisol plasmático foram separados em quartis, foi interessante notar que grupos com maiores $(\mathrm{P}<0,05)$ níveis de cortisol apresentaram maiores $(\mathrm{P}<0,05)$ médias da nota de temperamento. Porém, a diferença na TC entre esses grupos de animais foi apenas numérica $(\mathrm{P}>0,05)$, de modo que a TC $(59 \%)$ das novilhas menos reativas (cortisol $=4,12 \pm 1,12 \mathrm{ng} / \mathrm{mL} ; \quad \mathrm{NTe}=3,2 \pm 0,6$; $\mathrm{n}=22)$ não diferiu $(\mathrm{P}>0,05)$ da $\mathrm{TC} \quad(41 \%)$ das mais estressadas (cortisol $=7,76 \pm 1,33 \mathrm{ng} / \mathrm{mL}$; $\mathrm{NTe}=3,82 \pm 0,79 ; \mathrm{n}=22$ ), conforme demonstrado na Tab. 2. Pela correlação de Pearson, utilizandose dados das novilhas submetidas à dosagem de cortisol no D9, correlações positivas significativas foram observadas entre cortisol e $\mathrm{NTe}$, no $\mathrm{D} 9(\mathrm{P}=0,0020$ e $\mathrm{r}=0,4562)$, e entre cortisol e NTe, no D11 ( $\mathrm{P}=0,0004$ e $\mathrm{r}=0,5128$ ). Porém, não foi observada correlação significativa entre cortisol e TC $(\mathrm{P}=0,67 \mathrm{r}=-0,65)$.

Em estudo recente, Cooke et al. (2017) avaliaram o efeito do temperamento de 953 vacas pluríparas lactantes da raça Nelore, com 50 dias pós-parto, submetidas a um protocolo de IATF semelhante ao do presente experimento. Os autores observaram maior $(\mathrm{P}<0,05)$ concentração de cortisol e tendência para menor $(\mathrm{P}=0,09)$ TC nos animais excitáveis/reativos em comparação aos animais mais calmos. Também foi observada maior perda gestacional e redução em alguns parâmetros de produtividade dos animais com temperamento mais sanguíneo (Cooke et al., 2017). No presente estudo, não foi observado efeito do nível de cortisol e/ou do temperamento sobre a TC, embora tenha sido observada redução numérica de $18 \%$ na TC das novilhas com cortisol >6ng/mL (NTe D11=3,9) em comparação ao grupo das novilhas com cortisol <6ng/mL (NTe D11=3,1). Salienta-se ainda que esses dados incluem apenas novilhas em que não foi observada dificuldade de inseminação (apenas animais DifIA1). 


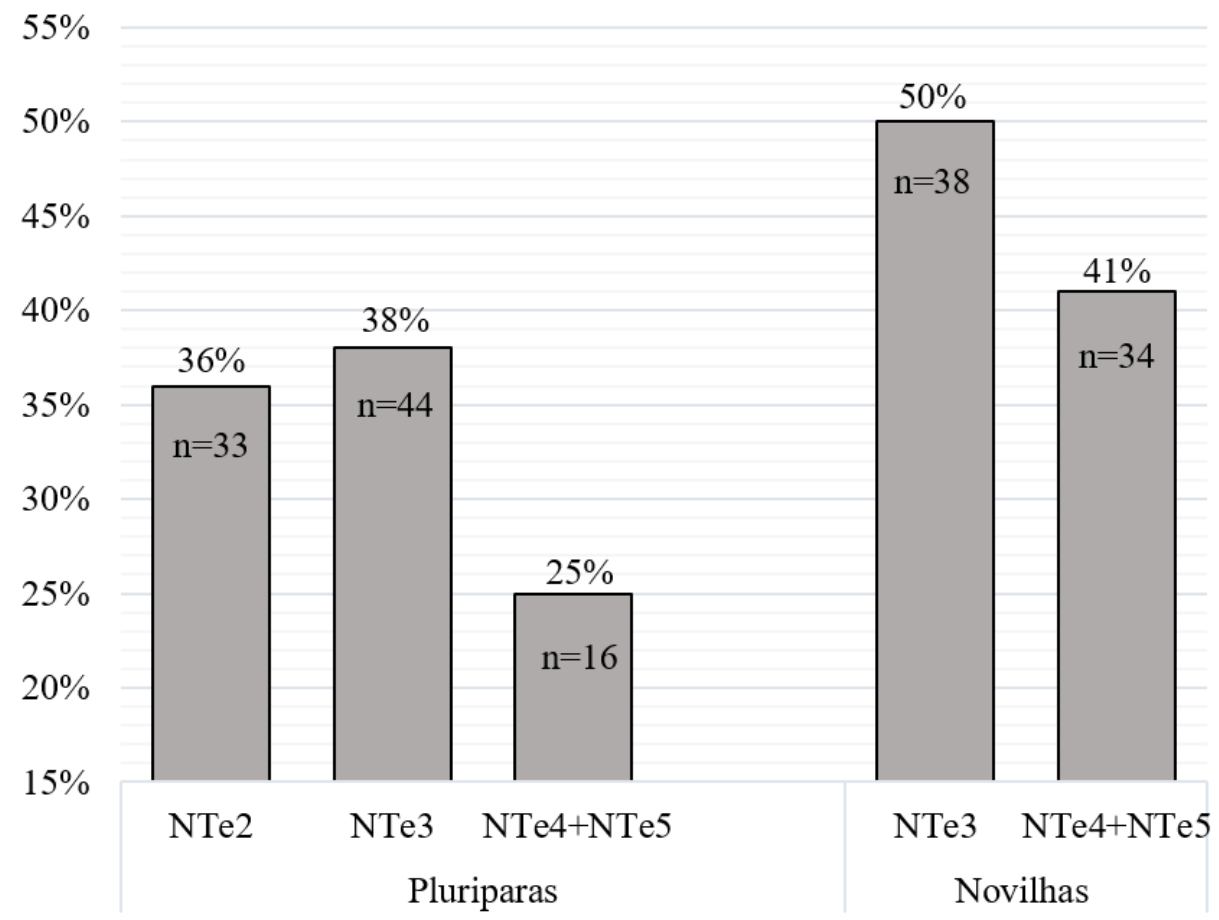

Figura 1. Taxa de concepção de acordo com a nota de temperamento (NTe) observada durante realização do protocolo de IATF; $(\mathrm{P}>0,05)$.

Tabela 2. Taxa concepção (TC) e média ( \pm desvio-padrão) da concentração plasmática de cortisol (ng/mL) no dia da remoção do implante de P4 (D9), da nota de reatividade/temperamento (NTe; 1 a 5) e do tempo de saída do brete (TSB; segundos) de novilhas Nelore $(n=44)$ submetidas ao mesmo protocolo de IATF. Os animais foram separados em quartil (Q), de acordo com a concentração de cortisol dosado por radioimunoensaio

\begin{tabular}{|c|c|c|c|c|c|c|c|c|}
\hline $\mathrm{N}$ & $\begin{array}{c}\text { Quartil } 1 \\
\text { (Q1) } \\
11\end{array}$ & $\begin{array}{c}\text { Quartil } 2 \\
\text { (Q2) } \\
11\end{array}$ & $\begin{array}{c}\text { Quartil } 3 \\
\text { (Q3) } \\
11\end{array}$ & $\begin{array}{c}\text { Quartil } 4 \\
\text { (Q4) } \\
11\end{array}$ & $\begin{array}{c}\text { Valor } \\
\mathrm{P}\end{array}$ & $\begin{array}{c}\mathrm{Q} 1+\mathrm{Q} 2 \\
22\end{array}$ & $\begin{array}{c}\mathrm{Q} 3+\mathrm{Q} 4 \\
22\end{array}$ & $\begin{array}{c}\text { Valor } \\
\mathrm{P}\end{array}$ \\
\hline $\begin{array}{l}\text { Valor no } \\
\text { quartil } \\
\text { (ng/mL) }\end{array}$ & $\begin{array}{c}(2,04 \mathrm{a} \\
4,31)\end{array}$ & $\begin{array}{c}(4,52 \mathrm{a} \\
5,87)\end{array}$ & $\begin{array}{c}(6,00 \mathrm{a} \\
7,55)\end{array}$ & $\begin{array}{l}(7,60 \mathrm{a} \\
10,03)\end{array}$ & & $(<6 \mathrm{ng} / \mathrm{mL})$ & $(>6 \mathrm{ng} / \mathrm{mL})$ & \\
\hline $\begin{array}{l}\text { Cortisol } \\
\text { (ng/mL) }\end{array}$ & $\begin{array}{l}3,24 \pm \\
0,84 \mathrm{a}\end{array}$ & $\begin{array}{l}5,00 \pm \\
0,48 \mathrm{~b}\end{array}$ & $\begin{array}{l}6,61 \pm \\
0,47 \mathrm{c}\end{array}$ & $\begin{array}{l}8,92 \pm \\
0,74 d\end{array}$ & $<0,001$ & $\begin{array}{l}4,12 \pm \\
1,12 \mathrm{a}\end{array}$ & $\begin{array}{l}7,76 \pm \\
1,30 \mathrm{~b}\end{array}$ & $<0,001$ \\
\hline $\begin{array}{l}\text { Taxa } \\
\text { concepcão }\end{array}$ & $64 \%$ & $55 \%$ & $36 \%$ & $45 \%$ & 0,3563 & $59 \%$ & $41 \%$ & 0,1885 \\
\hline $\begin{array}{l}\text { TSB no D9 } \\
\text { (s) }\end{array}$ & $\begin{array}{l}3: 32 \pm \\
0: 38 \mathrm{~A}\end{array}$ & $\begin{array}{c}2: 30 \pm \\
0: 41 \mathrm{AB}\end{array}$ & $\begin{array}{c}2: 35 \pm \\
0: 57 \mathrm{AB}\end{array}$ & $\begin{array}{l}2: 28 \pm \\
0: 48 B\end{array}$ & 0,0885 & $\begin{array}{c}02: 53 \pm \\
0: 43\end{array}$ & $\begin{array}{c}2: 27 \pm \\
0: 49\end{array}$ & 0,2242 \\
\hline NTe no D9 & $\begin{array}{l}3,09 \pm \\
0,62 \mathrm{a}\end{array}$ & $\begin{array}{c}3,23 \pm \\
0,61 \mathrm{ab}\end{array}$ & $\begin{array}{c}3,64 \pm \\
0,81 \mathrm{ab}\end{array}$ & $\begin{array}{l}4,01 \pm \\
0,77 \mathrm{~b}\end{array}$ & 0,0190 & $\begin{array}{l}3,16 \pm \\
0,60 \mathrm{a}\end{array}$ & $\begin{array}{l}3,82 \pm \\
0,79 b\end{array}$ & 0,0117 \\
\hline $\begin{array}{l}\text { TSB no } \\
\text { D11(s) }\end{array}$ & $\begin{array}{c}2: 52 \pm \\
0: 59\end{array}$ & $\begin{array}{c}2: 87 \pm \\
1: 21\end{array}$ & $\begin{array}{c}2: 67 \pm \\
0: 38\end{array}$ & $\begin{array}{c}2: 23 \pm \\
0: 33\end{array}$ & 0,1185 & $\begin{array}{c}2: 68 \pm \\
1: 09\end{array}$ & $\begin{array}{c}2: 30 \pm \\
0: 36\end{array}$ & 0,2836 \\
\hline $\begin{array}{l}\text { NTe no } \\
\text { D11 }\end{array}$ & $\begin{array}{l}3,12 \pm \\
0,48 \mathrm{a}\end{array}$ & $\begin{array}{l}3,04 \pm \\
0,72 \mathrm{a}\end{array}$ & $\begin{array}{l}3,82 \pm \\
0,51 \mathrm{~b}\end{array}$ & $\begin{array}{l}3,95 \pm \\
0,65 b\end{array}$ & 0,0124 & $\begin{array}{c}3,09 \pm \\
0,6 \mathrm{a}\end{array}$ & $\begin{array}{l}3,89 \pm \\
0,58 \mathrm{~b}\end{array}$ & 0,0002 \\
\hline
\end{tabular}

IATF: inseminação artificial em tempo fixo; D9: dia da remoção do implante de progesterona (P4); D11: dia da IATF;

a,b: letras minúsculas na mesma linha indicam diferença estatística $(\mathrm{P}<0,05)$;

A,B: letras maiúsculas na mesma linha indicam tendência estatística $(\mathrm{P}<0,10)$. 
Foi observada tendência para maior $(\mathrm{P}<0,10)$ TC nos animais em que não existiu dificuldade de IA (DifIA 1; TC $=42 \%, n=143$ ) em comparação aos animais que apresentaram moderada ou alta dificuldade de IA quando os dados de vacas e novilhas foram analisados em conjunto (DifIA 2 + DifIA3; $\mathrm{TC}=27 \%, \mathrm{n}=22$ ). Ao se compararem esses dois grupos, foi observado menor $(\mathrm{P}<0,001)$ tempo de IA para DifIA1 $(17: 31 \pm 06: 02 s)$ do que para DifIA2e3 (30:10 $\pm 15: 45 \mathrm{~s})$. Quando se separaram as duas categorias animal, a redução observada na TC foi apenas numérica (pluríparas: DifIA1 $=37 \%$, $\mathrm{n}=76$; $\quad$ DifIA2e3 $=29 \%, \quad \mathrm{n}=17$; novilhas: DifIA1 $=48 \%, \mathrm{n}=67$; DifIA2e3=20\%, n=5).

Nas fêmeas com maior dificuldade de inseminação, aumenta-se a chance de deposição da dose inseminante no(s) último(s) anel(éis) cervical(is) e não no corpo do útero. Em bovinos, há muito tempo não existem mais dúvidas sobre a vantagem da deposição de sêmen no corpo uterino em comparação à deposição na cérvix (Peters et al., 1984; Senger et al., 1988). De acordo com Peters et al. (1984), erros na inseminação convencional (no corpo do útero) são comuns, de modo que não é rara a inseminação intracervical profunda, a qual pode representar mais de $20 \%$ das tentativas de deposição do sêmen no corpo uterino. Em ovelhas, um dos fatores que mais afetam o sucesso da IA é justamente a inseminação de animais em que não é possível a completa transposição da cérvix, principalmente quando se utiliza sêmen congelado (Bicudo et al., 2005). Segundo Senger et al. (1988), uma das explicações mais plausíveis para quando se observa melhora da TC na inseminação intercornual de bovinos com sêmen congelado (quando comparada com a inseminação artificial convencional) é a redução da ocorrência de deposição seminal intracervical. Além disso, a deposição do sêmen dentro do útero reduz as perdas espermáticas por fagocitose durante a migração uterina, além de proporcionar um acréscimo na sobrevida do espermatozoide, em comparação com a inseminação intracervical (Senger et al., 1988). Por fim, não se deve descartar a possibilidade de que o maior tempo requerido para a realização das inseminações em que se observou maior dificuldade de IA possa interferir em alguns parâmetros espermáticos, dependendo do sêmen utilizado (Oliveira et al., 2012). Adicionalmente, uma interação entre elevada temperatura ambiente e aumento do intervalo da deposição de sêmen pode ocorrer devido a uma incubação prolongada do sêmen descongelado na palheta (DeJarnette et al., 2002).

Diante do exposto, o presente estudo demonstrou que as avaliações subjetiva (NTe) e numérica (TSB) do temperamento se relacionaram com o nível de estresse do animal. Portanto, o treinamento da mão de obra para manejo racional dos bovinos e a adoção de critérios de seleção em função de características da reatividade e/ou do temperamento animal devem ser utilizados em sistemas de criações extensivas, uma vez que alterações comportamentais dos animais geram alterações dos níveis séricos de cortisol.

Embora seja reconhecido que a minimização do estresse do gado (e das pessoas envolvidas no trabalho de campo) pode melhorar índices de produtividade e/ou de fertilidade de vacas de corte, os parâmetros de estresse avaliados no presente estudo não afetaram a taxa de concepção. Porém, menor fertilidade na IATF foi sugerida nos animais em que se observou maior dificuldade e/ou tempo necessário para se completar a IA.

\section{REFERÊNCIAS}

BAGE, R.; FORSBERG, M.; GUSTAFSSON, H.; LARSSON, B. Effects of ACTH-challenge on progesterone and cortisol levels in ovariectomised repeat breeder heifers. Anim. Reprod. Sci., v.63, p.65-76, 2000.

BARUSELLI, P.S.; REIS, E.L.; MARQUES, M.O. et al. The use of hormonal treatments to improve reproductive performance of anestrous beef cattle in tropical climates. Anim. Reprod. Sci., v.82-83, p.479-486, 2004.

BICUDO, S.D.; AZEVEDO, H.C.; SILVA MAIA, M.S. et al. Aspectos peculiares da inseminação artificial em ovinos. Acta Sci. Vet., v.33, p.127-130, 2005.

BÓ, G.A.; BARUSELLI, P.S Synchronization of ovulation and fixed-time artificial insemination in beef cattle. Animal, v.8, p.144-150, 2014. 
BURROW, H.M.; PRAYAGA, K.C. Correlated responses in productive and adaptive traits and temperament following selection for growth and heat resistance in tropical beef cattle. Livest. Prod. Sci., v.86, p.143-161, 2004.

COOKE, R.F.; BOHNERT, D.W.; MENEGHETTI, M. et al. Effects of temperament on pregnancy rates to fixed-timed AI in Bos indicus beef cows. Livest. Sci., v.142, p.108-113, 2011.

COOKE, R.F.; SCHUBACH K.M.; MARQUES R.S. et al. Effects of temperament on physiological, productive, and reproductive responses in beef cows. J. Anim. Sci., v.95, p.1-8, 2017.

DeJARNETTE J.M.; SHEPARD R.W.; KAPROTH M.T. et al. Effects of sequential insemination number after batch-thawing on conception rates of cryopreserved bovine semen: a review. In: TECHNICAL CONFERENCE ON ARTIFICIAL INSEMINATION AND REPRODUCTION, 19., 2002, Columbia. Proceedings... Columbia: [s.n.], 2002. p.102110.

DIAS, E.A.R.; ARRUDA, R.P.; VIDESCHI, R.A. et al. O uso de ECG influencia a taxa de concepção em vacas Nelore de diferentes condições corporais submetidas ao mesmo protocolo de IATF? Bol. Ind. Anim., v.70, p.215220, 2013.

DOBSON, H.; SMITH, R.F. What is stress, and how does it affect reproduction. Anim. Reprod. Sci., v.60-61, p.743-752, 2000.

FERRAZ JUNIOR, M.V.C.; PIRES, A.V.; BIEHL, M.V. et al. Comparison of two timed AI system schemes to synchronize estrus and ovulation in Nellore cattle. Theriogenology, v.86, p.1939-1943, 2016.

GRANDIN, T. Behavioral agitation during handling in cattle is persistent over time. Appl. Anim. Behav. Sci., v.36, p.1-9, 1993.

HEMSWORTH, P.H.; COLEMAN, G.J.; BARNETT, J.L.; BORG, S. Relationships between human-animal interactions and productivity of commercial dairy cows. J. Anim. Sci., v.78, p.2821-2831, 2000.
MACEDO, G.G.; ZÚCCARI, C.E.S.N.; ABREU, U.G.P. et al. Human-animal interaction, stress, and embryo production in $B o s$ indicus embryo donors under tropical conditions. Trop. Anim. Health Prod., v.43, p.1175-1182, 2011.

MAZIEIRO, R.R.D.; MARTIN, I.; MATTOS, M.C.C.; FERREIRA, J.C.P. Avaliação das concentrações plasmáticas de cortisol e progesterona em vacas Nelore submetidas a manejo diário ou manejo semanal. Vet. Zootec., v.19, p.366-372, 2012.

MENCHACA, A.; NUNEZ, R.; WIJMA, R. et al. How fertility can be improved in fixed-time AI programs in beef cattle. In: SYMPOSIUM ON ANIMAL REPRODUCTION, 10., 2013, Córdoba. Proceedings... Córdoba: [s.n.] 2013. p.103-134.

MENEGHETTI, M.; SÁ FILHO, O.G.; PERES, R.F.G. et al. Fixed-time artificial insemination with estradiol and progesterone for Bos indicus cows I: Basis for development of protocols. Theriogenology, v.72, p.179-189, 2009.

MESQUITA, F.S.; PUGLIESI, G.; SCOLARI, S.C. et al. Manipulation of the periovulatory sex steroidal milieu affects endometrial but not luteal gene expression in early diestrus Nelore cows. Theriogenology, v.81, p.861-869, 2014.

NARAYAN, E.; PARISELLA, S. Influences of the stress endocrine system on the reproductive endocrine axis in sheep. Ital. J. Anim. Sci., v.16, p.640-651, 2017.

OLIVEIRA, L.Z.; ARRUDA, R.P.; ANDRADE, A.F.C. et al. Effect of sequence of insemination after simultaneous thawing of multiple semen straws on conception rate to timed AI in Nelore cows. Theriogenology, v.78, p.1800-1813, 2012.

PARANHOS DA COSTA, M.J.R.; SANT'ANNA, A.C.; SILVA, L.C.M. Temperamento de bovinos Gir e Girolando: efeitos genéticos e de manejo. Inf. Agropecu., v.36, p.100-107, 2015.

PERES, R.F.G.; CLARO JÚNIOR, I.; SÁ FILHO, O.G. et al. Strategies to improve fertility in Bos indicus postpubertal heifers and nonlactating cows submitted to fixed-time artificial insemination. Theriogenology, v.72, p.681-689, 2009. 
PERRY, G.A.; SMITH, M.F.; ROBERTS, A.J. et al. Relationship between size of ovulatory follicle and pregnancy beef heifers. J. Anim. Sci., v.85, p.684-689, 2007.

PETERS, J.L.; SENGER, P.L.; ROSENBERGUER, J.L.; O'CONNOR, M.L. Radiographic evaluation of bovine artificial inseminating among professional and herdsmaninseminators using .5- and .25-ml french straws. J. Anim. Sci., v.59, p.1671-1683, 1984.

RUEDA, P.M.; SANT'ANNA, A.C.; VALENTE, T.S.; PARANHOS DA COSTA, M.J.R. Impact of the temperament of Nellore cows on the quality of handling and pregnancy rates in fixed-time artificial insemination. Livest. Sci., v.177, p.189-195, 2015.

SÁ FILHO, O.G.; VASCONCELOS, J.L.M. Treatments to optimize the use of artificial insemination and reproductive efficiency in beef cattle under tropical environments. Vet. Med. Intern., article ID 923053, p.1-10, 2011.

SANT'ANNA, A.C.; BALDI, F.S.; VALENTE, T.S. et al. Genetic associations between temperament and performance traits in Nellore beef cattle. J. Anim. Breed. Genet., v.132, p.4250, 2015.
SARTORI, R.; GIMENES, L.U.; MONTEIRO, P.L.Jr. et al. Metabolic and endocrine differences between Bos taurus and Bos indicus females that impact the interaction of nutrition with reproduction. Theriogenology, v.86, p.32-40, 2016.

SENGER, P.L.; BECKER, W.C.; DAVIDGE, S.T. et al. Influence of cornual insemination on conception in dairy cattle. J. Anim. Sci., v.60, p.3010-3016, 1988.

SILVEIRA, I.D.B.; FISCHER, V.; SOARES, G.J.D. Relação entre o genótipo e o temperamento de novilhos em pastejo e seu efeito na qualidade da carne. Rev. Bras. Zootec., v.35, p.519-526, 2006.

VALENTE, T.S.; SANT'ANNA, A.C.; BALDI, $\mathrm{F}$. et al. Genetic association between temperament and sexual precocity indicator traits in Nellore cattle. J. Appl. Genet., v.56, p.349$354,2015$. 